\title{
Zivilgesellschaftliche Konstruktionen politischer Realität
}

\author{
Eine vergleichende Analyse zu Themen und Nachrichtenfaktoren in politischen Weblogs \\ und professionellem Journalismus
}

Christiane Eilders / Sebastian Geißler / Michael Hallermayer / Michael Noghero / JanMathis Schnurr

Durch die neuen Nutzungsweisen des Internets, vor allem des Web 2.0, können sich zivilgesellschaftliche Akteure ohne Umweg über die journalistische Vermittlung in den politischen Diskurs einbringen. Damit dürfte sich nicht nur der Umfang der verfügbaren politischen Informationen sowie die Anzahl und Vielfalt der Kommunikatoren vermehren, sondern auch die politischen Inbalte verändern. Die Beiträge der zivilgesellschaftlichen Akteure, die hier als neue Öffentlichkeitssprecher auftreten, werden häufig als Bürgerjournalismus begriffen. Es wird angenommen, dass die Bürgerjournalisten politische Themen und Ereignisse nach anderen Kriterien auswäblen und darstellen als professionelle Journalisten und dass die resultierenden Inhalte daber nicht so sebr die mediale Auswabl- und Darstellungslogik als vielmehr die Relevanzwabrnebmung der Zivilgesellschaft widerspiegeln. Diese Annabme wird inhaltsanalytisch anhand einer vergleichenden Studie politischer Weblogs und Nachrichten in etablierten Massenmedien überprüft. Im Mittelpunkt stehen dabei Themen und Nachrichtenfaktoren. Die Befunde zeigen, dass Bürgerjournalisten zwar eine größere Vielfalt an Themen ansprechen, sich allerdings in Bezug auf die Nachrichtenfaktoren nur teilweise von den professionellen Journalisten unterscheiden.

Schlagwörter: Politische Kommunikation, Bürgerjournalismus, Weblogs, Web 2.0, Nachrichtenwertforschung, Zivilgesellschaft

\section{Hintergrund und Fragestellung}

Mit der Etablierung des Web 2.0 haben sich die direkten Beteiligungsmöglichkeiten der Bürger am politischen Diskurs enorm erweitert. Nicht nur die politischen Akteure können sich in eigenen Netzauftritten direkt an die Bürger wenden, auch die Bürger selbst sind weniger auf die Vermittlungsleistung der Massenmedien angewiesen. Sie können als Nutzer ihre eigenen Inhalte generieren (User-generated-Content) und ihre Sicht der politischen Realität einem größeren Publikum mitteilen. Damit treten sie in Konkurrenz zum professionellen Journalismus, dessen Leistungen in Bezug auf die politische Informationsvermittlung, die Kritik und Kontrolle des politischen Systems und die Orientierung des Publikums häufig in Frage gestellt werden (z. B. Neidhardt 1994, Ferree et al. 2002, Page 1996). So wird argumentiert, dass die professionellen Selektions- und Präsentationslogiken, die häufig als „Medienlogik“ zusammengefasst werden, diesen normativen Funktionszuweisungen im Wege stehen. Die Medienlogik begünstige die Relevanzzuweisungen und Sichtweisen der politischen Entscheidungseliten und vernachlässige im Gegenzug die Themeninteressen, Problemwahrnehmungen und Anliegen der Bürger.

Bei der Untersuchung der Medienlogik hat sich vor allem in Europa die Nachrichtenwertforschung als prominentester Forschungsansatz durchgesetzt (Eilders 2006). Die Nachrichtenwertforschung geht davon aus, dass Journalisten Ereignissen aufgrund be- 
stimmter Merkmale besonders hohe Relevanz zuweisen und diese daher eine höhere Publikationschance besitzen (z. B. Schulz 1976). Die entsprechenden Merkmale werden als Nachrichtenfaktoren bezeichnet. Sie leiten die Nachrichtenauswahl und die Präsentation von Nachrichten an. Da sie so die journalistische Konstruktion politischer Realität bestimmen, lassen sie sich als Konstruktionskriterien begreifen. Besonders wirksame Nachrichtenfaktoren sind etwa Reichweite (Anzahl der Betroffenen), Schaden bzw. Negativismus, Nutzen, Dauer des Ereignisses, Faktizität (Ereignishaftigkeit), Elitebezug (Prominenz und Einfluss von Akteuren), Personalisierung und Konflikt.

Als Gegenmodell zur professionell-journalistischen Politikberichterstattung wird häufig auf die politischen Weblogs verwiesen, ${ }^{1}$ also auf diejenigen Formate des Usergenerated-Content, in denen Nutzer frei von der Medienlogik ihr eigenes Bild der politischen Realität konstruieren. Nun ist bekannt, dass die Nachrichtenauswahl und -präsentation entlang von Nachrichtenfaktoren keineswegs nur die journalistische Realitätskonstruktion anleitet. Vielmehr lassen sich Nachrichtenfaktoren auf allgemeinmenschliche und nicht auf Journalisten beschränkte Wahrnehmungskriterien zurückführen (Eilders 1997, Eilders/Wirth 1999, Shoemaker/Cohen 2006, Fretwurst 2008). Auch die Realitätskonstruktion der nicht-journalistischen Nutzer dürfte demnach nicht ganz frei von dieser Logik sein. Nachdem allerdings davon auszugehen ist, dass Nachrichtenfaktoren im professionellen Journalismus zusätzlich durch Sozialisation und Ausbildung verankert sind, dürften die professionellen stärker als die nicht-professionellen Beiträge von Nachrichtenfaktoren geprägt sein.

Über den Nutzen von Weblogs für den politischen Diskurs ist eine kontroverse Debatte entbrannt, die sich nicht nur in der skeptischen gegenseitigen Beobachtung von professionellen Journalisten und Bloggern niederschlägt, sondern auch in der wissenschaftlichen Auseinandersetzung (zusammenfassend Neuberger et al. 2009, Engesser 2008). In dieser Debatte geht es v. a. darum, ob politische Weblogs als (Bürger- ${ }^{2}$ ) Journalismus begriffen werden können, oder ob es sich grundsätzlich nicht um Journalismus handelt, wenn Beobachtungen und Einschätzungen politischer Realität jenseits professionell-redaktioneller Kontexte und Qualitätskriterien veröffentlicht werden. Der vorliegende Beitrag knüpft an diese Debatte ${ }^{3}$ an. Er fragt jedoch nicht nach den Entstehungskontexten der jeweiligen Realitätskonstruktionen, sondern fokussiert auf deren Resultate. Ziel ist es, den inhaltlichen Beitrag von Weblogs zum politischen Diskurs zu bestimmen.

Um die Erwartungen in Bezug auf den inhaltlichen Beitrag von politischen Weblogs zu spezifizieren, nehmen wir die Rolle der Bürger bei der politischen Informationsvermittlung in den Blick und greifen dabei auf das gut ausgearbeitete Konzept der Zivilge-

1 Beispielhaft dazu Engesser/Wimmer (2009). Hier wird anhand ausgewählter Fallbeispiele diskutiert, inwiefern sich in den partizipativen Formaten des Web 2.0 Ansätze zu einer Etablierung von Gegenöffentlichkeit finden lassen.

2 Obgleich es sich begrifflich anbieten würde, haben wir für die Weblogs bewusst nicht den Begriff „Laienjournalismus“ gewählt, weil sich teilweise auch professionelle Journalisten außerhalb ihrer Berufstätigkeit als Blogger betätigen (besonders prominent: Stefan Niggemeier), die also nicht als Laien bezeichnet werden können. Die professionellen Journalisten agieren hier außerhalb ihres redaktionellen Kontextes allerdings stärker in der Rolle von Bürgern und weniger in ihrer Berufsrolle.

3 Vgl. beispielhaft dazu die Debatte zwischen Miriam Meckel und Stefan Niggemeier in FAZ.net am 12.05.2009 (Meckel 2009, Niggemeier 2009). Hier weist Meckel darauf hin, dass lediglich der professionelle Journalismus eine kontinuierliche und systematische Umweltbeobachtung leiste, während Niggemeier auf entsprechende Fehlleistungen der etablierten Massenmedien verweist. 
sellschaft zurück (Habermas 1998, Heming 1997, Klein 2008). Der Begriff umfasst nach Habermas das aus der staatlichen und wirtschaftlichen Sphäre einerseits und der Privatsphäre andererseits hervortretende Publikum von Bürgern, das sich aktiv am öffentlichen Diskurs beteiligt und damit auf die institutionalisierte Meinungs- und Willensbildung Einfluss nimmt (Habermas 1998: 444). Mit Blick auf die Artikulation der Bürger in politischen Weblogs ist das Konzept der Zivilgesellschaft für die Analyse von Bürgerjournalismus damit eine besonders geeignete theoretische Referenz (vgl. dazu auch Eilders 2010) und fundiert unser Verständnis von Bürgerjournalismus. In welchem Maße sich die Inhalte politischer Weblogs einerseits und die professionelle Berichterstattung andererseits unterscheiden, soll in diesem Beitrag untersucht werden. Nur wenn in Weblogs die politische Realität anders als in den etablierten Massenmedien konstruiert wird, kann von einer substanziellen inhaltlichen Erweiterung des politischen Diskurses ausgegangen werden.

Trotz der öffentlichkeitstheoretischen Brisanz und des schnell zunehmenden Interesses am Web 2.0 in den vergangenen Jahren ist der bisherige empirische Forschungsstand zu Weblog-Inhalten im deutschsprachigen Raum als äußerst dürftig zu bezeichnen. Die aktuelle Forschung setzt kaum bei den Inhalten, sondern vor allem bei den Akteuren der Blogosphäre an und gibt damit kaum Aufschluss über die Konstruktionsmechanismen des Bürgerjournalismus. Unsere Inhaltsanalyse leistet einen ersten Beitrag zur Schließung dieser Forschungslücke, indem sie die inhaltliche Leistung von politischen Weblogs und professioneller Berichterstattung untersucht. Sie hat explorativen Charakter, da hier erstmalig die Inhalte polischer Weblogs systematisch mit professionell journalistischer Berichterstattung verglichen werden und der geringe Umfang des empirischen Materials kaum belastbare Aussagen zulässt. Die Befunde geben jedoch einen ersten Eindruck der inhaltlichen Besonderheiten. Wir gehen in zwei Schritten vor, um sowohl einen breiten Überblick über die inhaltlichen Profile als auch tiefere Einblicke in die jeweiligen Konstruktionsmechanismen der Beiträge zu erhalten. Im ersten Schritt werden die Themenpräferenzen und die geographischen Schwerpunkte der Beiträge verglichen, während im zweiten Schritt in einer vertiefenden Analyse zu sieben ausgewählten Themen die Nachrichtenfaktoren untersucht werden, die die Beiträge in den verschiedenen Medienangeboten kennzeichnen.

\section{Weblogs als Bürgerjournalismus}

\subsection{Bürger-oder Pseudo-Journalismus: Abgrenzungskriterien von Journalismus}

Nur wenn sich Weblogs als spezifische Form von Journalismus begreifen lassen, lässt sich eine Kontrastierung von Weblogs und professionellem Journalismus rechtfertigen. Zunächst ist also zu klären, ob Weblogs den Kriterien von Journalismus entsprechen. Die Schwierigkeiten bei der Definition von Journalismus sind hinlänglich bekannt. Je nachdem, vor welchem allgemein gesellschaftstheoretischen und spezifisch kommunikationswissenschaftlichen Hintergrund welche konkrete Definition gewählt wird, kommen unterschiedliche Abgrenzungskriterien ins Spiel (Löffelholz 2004). Noch schwieriger wird es, wenn es darum geht, die Journalismuskonzepte auf die neuen Angebote im Internet anzuwenden. Neuberger betont, dass das herkömmliche Verständnis von Journalismus den Phänomenen von Internetöffentlichkeit kaum noch gerecht wird, da der Journalismusbegriff in der Regel mit den Merkmalen Berufstätigkeit und Redaktionskontext assoziiert wird (Neuberger 2008). Quandts Zusammenschau von Journalismusdefinitionen bestätigt das (2004: 452). Wenn also die Online-Kommunikation nicht per se ausgeschlossen werden soll, sind die Produktionskontexte als Abgrenzungskrite- 
rium wenig geeignet. Auch Kriterien, die sich aus dem Verständnis von Massenkommunikation herleiten (so etwa das disperse Publikum als Adressat), greifen nicht, wenn es um Online-Angebote geht, da diese ja häufig eben nicht auf ein Massenpublikum zugeschnitten sind. Der Bezug auf Merkmale der Massenkommunikation schließt also $\mathrm{zu}$ viele Angebote aus der Journalismus-Definition aus. Umgekehrt werden zu viele Angebotstypen eingeschlossen, wenn die Definition am Tätigkeitsprofil der Aussagenproduzenten festgemacht würde. Schließlich können die Tätigkeiten im Rahmen von Content-Produktion für ein Unternehmen einerseits und in der professionell-journalistischen Produktion andererseits identisch sein (Quandt 2004: 453). Das gilt auch für das Verfassen oder die Bereitstellung von Inhalten in Weblogs, da diese Tätigkeiten ebenfalls im Wesentlichen Beobachten, Auswählen und Schreiben umfassen.

Der Zugang über die Produktionskontexte oder die Publikumsorientierung der Akteure einerseits und ihre Tätigkeiten andererseits erweist sich damit als wenig fruchtbar. Neuberger argumentiert, dass die häufig zum Zwecke der Abgrenzung angeführten Merkmale von Journalismus keinesfalls notwendige Merkmale darstellen und damit ungeeignet für eine Definition seien. Zielführend ist seiner Ansicht nach allein eine Bestimmung des Journalismus über seine gesellschaftlichen Funktionen (Neuberger 2008: 19). Die Vermittlungsleistung von Journalismus stellt dabei das zentrale Konzept dar. Der Journalismus vermittle zwischen den verschiedenen Teilen der Gesellschaft, indem er die Umwelt beobachte und diese Beobachtungen der Gesellschaft zur Verfügung stelle. Die Leistung könne dabei sinnvoll lediglich inhaltsanalytisch erfasst werden. Wenn es also um den Status von Weblogs als Journalismus gehe, seien entsprechende inhaltsanalytische Studien von zentraler Bedeutung. Wichtige Indikatoren für die Vermittlungsleistung könnten dabei etwa Themenrelevanz und Themenvielfalt seien (Neuberger 2008: 28).

Aus den gesellschaftlichen Funktionen lassen sich weitere Ansprüche an die zu vermittelnden Inhalte ableiten. Weitgehend konsensfähig ist dabei die Auffassung, dass von Journalismus nur in dem Maße gesprochen werden kann, in dem dabei Themen von allgemeiner Relevanz, Aktualität und Faktizität behandelt werden (Scholl/Weischenberg 1998: 78). Dem Kriterium der allgemeinen Relevanz entsprechen politische Medienangebote in hohem Maße. In Bezug auf die Frage, ob Weblogs als Produkte von Journalismus betrachtet werden können, wird daher immer wieder der Politikgehalt als Abgrenzungskriterium von Journalismus verwendet. Der unstrittig geringe politische Gehalt der Nutzer-generierten Inhalte in Deutschland (z.B. Neuberger et al. 2007) deutet nach Ansicht vieler Beobachter darauf hin, dass Weblogs nicht als Journalismus verstanden werden können. Politik kommt in der deutschen Blogosphäre in der Tat keine zentrale Bedeutung zu, der Umkehrschluss gilt indessen nicht. Für die Politik ist nämlich die Blogosphäre durchaus von Bedeutung. Hier geht es schließlich nicht um die relative Häufigkeit politischer und nicht-politischer Weblogs, sondern darum, dass eine kritische Masse überschritten ist, so dass man von einem wahrnehmbaren Einflussfaktor im politischen Diskurs ausgehen kann. Angesichts der durch zahlreiche Studien belegten Nutzung von politischen Weblogs als relevante Quellen durch Journalisten, politische Akteure und Bürger kann daran kein Zweifel bestehen (Neuberger et al. 2009: 174 und 184, Engesser/Wimmer 2009). Wenngleich nicht die Gesamtheit aller Weblogs als journalistisches Angebot begriffen werden kann, können doch die politischen Weblogs als Journalismus im weitesten Sinne aufgefasst werden. In dem Maße, in dem politischen Weblogs eine Orientierung an der Vermittlungsfunktion von Journalismus zugeschrieben werden kann und sie sich mit Themen von allgemeiner Relevanz, Aktualität und Faktizität befassen, treten sie jedenfalls in Konkurrenz zum professionellen Journalis- 
mus und können hinsichtlich ihres inhaltlichen Beitrags zum öffentlichen Diskurs mit dem professionellen Journalismus verglichen werden. Inwiefern diese Form von Journalismus als Bürgerjournalismus begriffen werden kann und was diesen kennzeichnet, ist Gegenstand des folgenden Abschnitts.

\subsection{Zivilgesellschaftliche Perspektiven im Bürgerjournalismus}

Die öffentlichkeitstheoretische Perspektive auf politische Weblogs als Bürgerjournalismus rückt den ersten Teil des Begriffs in den Vordergrund. Hier geht es um die Frage, ob die Sichtweisen und Anliegen der Bürger in den politischen Diskurs eingebracht werden, ob also in den Beiträgen der Nutzer zivilgesellschaftliche Perspektiven eingenommen werden, die möglicherweise vom professionellen Journalismus vernachlässigt werden. Die Bedeutung der zivilgesellschaftlichen Perspektive ergibt sich aus den Konzepten politischer Öffentlichkeit. Dabei ist auch hier die Vermittlungsfunktion Drehund Angelpunkt der Überlegungen.

Öffentlichkeit wird als Kommunikationssystem beschrieben, das zwischen politischer Entscheidungsebene und Bürgern vermittelt. Dabei kommen verschiedene Akteure als Öffentlichkeitssprecher zu Wort, deren Beiträge als „Input“ im System Öffentlichkeit zum Produkt „öffentliche Meinung“ als „Output“ verarbeitet werden (zusammenfassend Eilders 2008: 32ff.). Das Kommunikationssystem Öffentlichkeit erfüllt in diesem Modell die gesellschaftlichen Funktionen Transparenz, Validierung und Orientierung, indem es offen für verschiedene Interessen ist, die Sprecherbeiträge sich in der Auseinandersetzung mit anderen Beiträgen vor Publikum bewähren müssen, und das Publikum die so validierten Themen und Meinungen als akzeptable Alternativen wahrnimmt. ${ }^{4}$ In dem Maße, in dem dabei möglichst viele verschiedene Gesellschaftssegmente $\mathrm{zu}$ Wort kommen, also nicht nur die politischen Eliten, sondern auch zivilgesellschaftliche Akteure ihre Sichtweisen formulieren, verbessert sich die Qualität und Belastbarkeit der resultierenden Politikentscheidungen. ${ }^{5}$ Der Inklusion der Zivilgesellschaft im Diskurs kommt dabei deswegen eine so große Bedeutung zu, weil zivilgesellschaftlichen Akteuren eine besondere Problemsensibilität und damit ein Gespür für neue Themen zugeschrieben wird (Klein 2008, Heming 1997).

Während Einigkeit darüber besteht, dass ein großer Teil des öffentlichen Diskurses von den Massenmedien getragen wird, erweist sich eine Übertragung der Ansprüche auf die massenmediale Kommunikation als problematisch. So wird häufig in Zweifel gezogen, dass unter den Bedingungen der modernen Massenkommunikation gleiche Zugangschancen zur Öffentlichkeit bestehen (etwa Neidhardt 1994: 20f.). Die Aufmerksamkeitsökonomie der Massenmedien sowie die Verarbeitungsmechanismen sind durch eine ausgeprägte Selektivität bestimmt. Durch die Orientierung an Nachrichtenfaktoren werden bestimmte Gesellschaftssegmente und Realitätsaspekte systematisch ausgeschlossen. Der Auswahllogik der Medien entsprechen v. a. Akteure des politischen Zentrums, während die an der Peripherie angesiedelten zivilgesellschaftlichen Akteure nur selten Zugang zur Medienöffentlichkeit erhalten und damit entsprechende Anliegen und Interessen kaum repräsentiert sind.

4 Vgl. auch das Konzept der „reflektierten öffentlichen Meinungen“ in Habermas” Anwendung des deliberativen Öffentlichkeitsmodells auf die Mediengesellschaft (Habermas 2008: 167).

5 Neben der Offenheit des Zugangs zum öffentlichen Diskurs ist das Resultat auch abhängig von der hier nicht untersuchten Fairness und dem gegenseitigen Respekt der Sprecher sowie der Rationalität des Diskurses (vgl. Habermas 2008). 
Mit den erweiterten Beteiligungsmöglichkeiten am politischen Diskurs durch Weblogs haben sich nun die Bedingungen öffentlicher medial-vermittelter Kommunikation entscheidend verändert. Wesentlich ist dabei die Verringerung der Zugangsbarrieren zur Öffentlichkeit durch die Aufhebung der Trennung von Kommunikator und Rezipient im Konzept des „produsers“ (Bruns 2007). Die Zukunftsszenarien fokussieren auf die Demokratisierung des Diskurses und die durch die prinzipiell mögliche Partizipation verbesserte Deliberation politischer Entscheidungen. In Bezug auf die inhaltlichen Veränderungen des Diskurses, die mit der substanziellen Erweiterung und Pluralisierung des Sprecherspektrums erwartet werden, sind v. a. die Themen hervorzuheben. So wird angenommen, dass der Diskurs durch das neue Sprecherensemble die zivilgesellschaftlichen Anliegen und Sichtweisen besser reflektiert (Koopmans/Zimmermann 2003, Rucht et al. 2008, Morrisett 2003). ${ }^{6}$ Weiter wird erwartet, dass die Sprecherbeiträge nicht den Konstruktionskriterien des professionellen Journalismus folgen, sondern die „produsers“ neue Auswahl- und Darstellungsweisen entwickeln (Koopmans/Zimmermann 2003, Marschall 1997, Dahlberg 2001 und 2007). In welchem Maße Nachrichtenfaktoren dabei auch - wie oben erwähnt - die zivilgesellschaftlichen Realitätskonstruktionen beeinflussen, wird im Weiteren zunächst in Bezug auf den Forschungsstand zu Weblogspezifischen Realitätskonstruktionen und dann in Bezug auf unsere eigene empirische Studie zu prüfen sein.

\section{Empirische Befunde zu politischen Weblogs}

\subsection{Motivlage und Selbstverständnis von Bloggern}

Die Debatte zur Erweiterung des politischen Diskurses durch Weblogs hat zunächst weitgehend auf empirische Befunde verzichtet, und auch heute stehen die vielen theoretischen Arbeiten in deutlichem Gegensatz zum Mangel an soliden empirischen Befunden. Trotz der im Vergleich zum englischen Sprachraum noch geringeren Zahl empirischer Studien konzentrieren wir uns auf die Forschung im deutschen Sprachraum und betrachten dort v. a. Arbeiten zu zivilgesellschaftlich betriebenen Weblogs zum allgemeinen politischen Geschehen. ${ }^{7}$ Die Mehrzahl der einschlägigen Studien setzt nicht bei den konkreten Inhalten an, sondern befasst sich mit den Beteiligten des Diskurses. Der methodische Zugang erfolgt dementsprechend meist über Befragungen von Bloggern und Blognutzern sowie im Fall von Netzwerkanalysen über technisch generierte Daten. Diese Schwerpunkte spiegeln sich auch in den entsprechenden Überblicksartikeln und Sammelbänden zum Web 2.0. ${ }^{8}$

In Bezug auf typisch journalistische Motivlagen ergeben sich in verschiedenen Studien widersprüchliche Hinweise. In der Studie von Schmidt und Kollegen (Schmidt/ Wilbers 2006) werden überwiegend selbstbezogene Gründe für das Betreiben von Weblogs genannt (Spaß, gerne schreiben, Ideen für sich selbst festhalten). Entsprechend geben die meisten an, v. a. Berichte, Episoden und Anekdoten aus dem Privatleben zu veröf-

6 Dieser Optimismus bleibt nicht unwidersprochen: So machen pessimistische Beobachter mit Verweis auf die ungleiche Verteilung von kognitiven, motivationalen und materiellen Ressourcen auf eine anhaltende Exklusion bestimmter Bevölkerungssegmente aufmerksam (Neuberger 2003, Donges 2000, Hamelink 2000).

7 Auch in der einschlägigen internationalen Forschung sind uns allerdings keine Studien bekannt, die unserem Kriterium der zivilgesellschaftlichen Trägerschaft entsprechen.

8 In Bezug auf die deutsche Blogosphäre geben die Synopse von Neuberger, Nuernbergk und Rischke (2007 und 2009), der von Zerfaß, Welker und Schmidt (2008) herausgegebene Zweibänder sowie der Band von Quandt und Schweiger (2008) einen guten Überblick. 
fentlichen. Wenngleich immerhin 41 Prozent angeben, Kommentare zu aktuellen politischen Themen zu verfassen, werden nur selten Motive genannt, die auf die Funktionen von Weblogs für die politische Öffentlichkeit verweisen. Armborst (2006) identifiziert dagegen in seiner Untersuchung etwas ausgeprägtere journalistische Motivlagen. Dazu passt auch sein Befund, dass Blogger sich stark journalistischen Normen verpflichtet fühlen.

Die widersprüchlichen Ergebnisse (vgl. auch Neuberger et al. 2007: 110) sind typisch für den Forschungsstand zur Blogosphäre. Sie ergeben sich zum großen Teil aus der mangelnden Unterscheidung zwischen den verschiedenen Arten von Weblogs. Fokussiert man nämlich auf diejenigen Blogger, die sich mit politischen Themen auseinandersetzen, ergibt sich ein stimmigeres Bild. Schmidt und Kollegen (Schmidt et al. 2006) haben in einer Analyse der „Politblogger“ typische Charakteristika herausgearbeitet. Im Vergleich zur Gesamtheit der Befragten sind hier Männer und höher Gebildete deutlich überrepräsentiert. In einer von uns durchgeführten Sekundäranalyse der Daten aus der Weblog-Befragung durch Schmidt und seine Kollegen ${ }^{9}$ zeigt sich, dass die „Politblogger" sich außerdem durch einen etwas höheren Anspruch an den politischen Diskurs auszeichnen. Politblogger schätzen die Möglichkeit, „etwas zu lesen, was ich aus anderen Medien nicht erfahre“ (66,4\% vs. $52 \%)$, etwas höher ein und ihr Interesse an einer objektiv-ausgewogenen Diskussion von Themen (22\% vs. 18,8\%) sowie an der persönlichen Meinung der Autoren ist größer (82,5\% vs. 70,9\%).

\subsection{Strukturelle und inhaltliche Merkmale von Weblogs}

Auch mit der instruktiven Spezifizierung des journalismusnahen Selbstverständnisses von Politbloggern bleibt die empirische Evidenz hinsichtlich eines wesentlichen Beitrags politischer Weblogs zum politischen Diskurs unzureichend. Es liegt nahe, die Frage nach der Diskurserweiterung durch Weblogs nicht über die Charakteristika der Blogger weiterzuverfolgen, sondern sie stärker unter inhaltlicher Perspektive zu untersuchen. Zur Klassifikation verschiedener Blogtypen liegt eine Reihe von Studien vor (z. B. Hesse 2008, Berendt et al. 2008, Kunz 2006, zitiert nach Trepte et al. 2008: 511). Über die selektive Aufmerksamkeit für bestimmte Themen und Ereignisse ist hier jedoch wenig zu erfahren. ${ }^{10}$ Weiterführend sind Studien über die Verlinkung in der deutschen Blogosphäre, da sich hier jedenfalls indirekt Hinweise auf die selektive Aufmerksamkeit der Weblogs ergeben. Die Befunde deuten darauf hin, dass in großem Umfang auf die massenmediale Berichterstattung Bezug genommen wird (Berendt et al. 2008: 92, Schmidt 2009, zusammenfassend Neuberger et al. 2007: 110). Eine stark von den Massenmedien abweichende Agenda in politischen Weblogs ist auf der Basis dieser Befunde kaum zu

9 Wir bedanken uns dafür bei Jan Schmidt, der uns die Daten freundlicherweise verfügbar gemacht hat.

10 Auch in internationalen Studien geht die inhaltliche Charakterisierung kaum über eine grobe Kategorisierung verschiedener Blogtypen hinaus (Herring et al. 2004, Papacharissi 2007). Etwas stärker geraten die Weblog-Inhalte in der US-Studie von Lenhart und Fox (2006) in den Blick. Hier rangieren „Politik und Regierung“ an zweiter Stelle der angebotenen Themengebiete. Ausnahmen sind weiterhin Arbeiten zu den thematisch fokussierten „Warblogs“ (z. B. Tremayne et al. 2006) und Wahlkampfblogs (z. B. Williams et al. 2005, Trammel 2006), wobei hier politische Akteure und nicht die Zivilgesellschaft als Blogger im Vordergrund stehen. 
erwarten. ${ }^{11}$ Allerdings ist vorstellbar, dass Blogger zusätzliche Themen aufgreifen und etwas andere Akzente in der Darstellung setzen.

In Bezug auf die Erfassung konkreter Themen lassen sich für die deutsche Blogosphäre bislang nur zwei einschlägige Studien ausmachen. Berendt, Schlegel und Koch (2008) untersuchen neben der Verlinkung auf massenmediale Quellen in einer technischen, auf Worthäufigkeiten basierenden Analyse deutschsprachiger und englischsprachiger Weblogs neben Akteurs- und Ortsnennungen, welche Themenbereiche in den Weblogs angesprochen werden. Hier ergeben sich erste Hinweise auf die Aufmerksamkeitsstruktur von Weblogs. In den untersuchten Weblogs dominieren Beiträge zu Kollektivakteuren aus dem politischen Zentrum (CDU, Bundestag, Bundesregierung, SPD), zu Demokratie und Terror sowie zu Ereignissen im Irak und in Afghanistan. Demnach scheinen innerparteiliche Auseinandersetzungen sowie ausgewählte außenpolitische Politik-Bereiche besondere Beachtung in der Blogosphäre zu generieren (Berendt et al. 2008: 88).

Einschlägig ist ferner die inhaltsanalytische Studie von Unger (2005). Unger erfasst, welche Themenbereiche eine Stichprobe deutschsprachiger Weblogs ${ }^{12}$ inhaltlich abdeckt. Er stellt fest, dass „öffentlich relevante“ Themen sowie selbstreferenzielle Diskussionen über die Blogosphäre selbst dominieren. Der Anteil „öffentlich relevanter Themen“ ist dabei lediglich etwas höher als der Anteil der selbstreferenziell auf die Blogosphäre bezogenen Beiträge. Unger unterscheidet zwischen Politik, Wirtschaft, Kultur, Medien, Informationstechnologie, Wissenschaft und Recht. Die Themen Informationstechnik, Massenmedien und Kultur bestimmen dabei über 80 Prozent der Beiträge zu öffentlich relevanten Themen. Die politischen Beiträge spielen eine vergleichsweise unbedeutende Rolle. Sie befassen sich überwiegend mit innenpolitischen Fragen (Unger 2005: 80).

In Bezug auf die Frage nach Auswahl- und Präsentationslogiken von Bürgerjournalisten einerseits und professionellen Journalisten andererseits helfen auch die Befunde der beiden Themenanalysen nur bedingt weiter. Zum einen erlaubt der geringe Differenzierungsgrad der Themenkategorien kaum tiefere Einblicke in die spezifische Konstruktion der politischen Realität, zum anderen fehlt ein auf den gleichen Ereignishorizont bezogener Vergleich der bürgerjournalistischen und professionell-journalistischen Realitätskonstruktionen, um auf die Auswahl- oder Präsentationskriterien zu schließen. Entsprechend beweiskräftige Untersuchungen liegen bislang für den deutschen Sprachraum nicht vor.

Der Überblick über den Stand der Forschung zu politischen Weblogs im deutschen Sprachraum zeigt, dass zwar zahlreiche Arbeiten zur Systematisierung von Weblogs und anderen Formaten des User-generated Content, zu deren Nutzung sowie zu Selbstverständnis und Motivlage der Blogger vorliegen, in Bezug auf die konkreten Inhalte der Realitätskonstruktionen von Bürgerjournalisten und in Bezug auf die zugrundeliegenden Auswahl- und Präsentationskriterien jedoch noch erhebliche Defizite bestehen. Damit ist eine Forschungslücke markiert, die mithilfe der hier präsentierten Studie reduziert werden soll.

11 Eine Auswertung der international publizierten Blog-Beiträge pro Tag (Sifry 2006) bestätigt das: Blogs reagieren mit hoher Publikationsaktivität auf herausragende Ereignisse, die auch im Journalismus Aufmerksamkeit finden.

12 Ausgewählt wurden alle Blogs aus einer Liste der 100 URLs der am häufigsten in der deutschen Blogosphäre verlinkten Weblogs (www.blogstats.de), die mindestens vier eingehende Links aufwiesen. 
Die wesentlich umfangreichere US-amerikanische Forschung zur politischen Blogosphäre weist in dieser Hinsicht zwar ebenfalls Defizite auf (vgl. Fußnote 7), zumindest eine Studie kann jedoch als Orientierungspunkt dienen, wenngleich sie in Bezug auf die dort erfassten Weblogs nicht das Kriterium zivilgesellschaftlicher Trägerschaft erfüllt. So hat Xenos (2008) auf differenziertem Niveau die inhaltlichen Unterschiede zwischen der Wahlkampfberichterstattung in Weblogs und in den Massenmedien erfasst. Die Analyse bezieht sich zwar lediglich auf ein Einzelthema und berücksichtigt nur WeblogBeiträge, die auf Massenmedien verlinken ${ }^{13}$, ermöglicht jedoch durch den Medienvergleich Schlüsse auf die jeweiligen Konstruktionskriterien. Weiterführend ist vor allem sein Vorschlag, in Analogie zu den Nachrichtenfaktoren des professionellen Journalismus „Blogfaktoren“ zu identifizieren. Mit der Gegenüberstellung von Nachrichten- und Blogfaktoren könnten die Auswahl- und Darstellungskriterien in Weblogs und im professionellen Journalismus neu konzeptionalisiert werden (Xenos 2008: 501). Ob im Bürgerjournalismus tatsächlich eigene Blogfaktoren wirksam sind oder auch dort Nachrichtenfaktoren die Konstruktion der Realitätsbilder bestimmen, ist die zentrale Frage unserer empirischen Studie.

\section{Annahmen und Untersuchungsdesign}

Wie bereits von Neuberger (2008) ausgeführt, ist ein inhaltsanalytischer Zugriff erforderlich, um den Beitrag von Weblogs zum politischen Diskurs zu untersuchen, da nur über die inhaltlichen Charakteristika die jeweilige Vermittlungsleistung des professionellen Journalismus und des Bürgerjournalismus unabhängig von den Organisationsstrukturen erfasst werden kann (vgl. auch Abschnitt 2.1). Relevante inhaltliche Charakteristika sind die thematischen Schwerpunkte sowie die spezifischen Kriterien, nach denen die Realitätskonstruktionen erfolgen. Themen und Nachrichtenfaktoren stehen daher im Mittelpunkt der Untersuchung. In Bezug auf die Themenschwerpunkte gehen wir davon aus, dass die zivilgesellschaftlichen Realitätsbilder im Vergleich zur Berichterstattung des professionellen Journalismus stärker am lebensweltlichen Nahraum ausgerichtet sind. Nachdem allerdings bekannt ist, dass Blogger die Berichterstattung der Medien kritisch beobachten und kommentieren, dürfte sich das massenmediale Themenspektrum nichtsdestoweniger in den Weblogs widerspiegeln, so dass keinesfalls zwei völlig getrennte Themenrepertoires in Weblogs und Massenmedien zu erwarten sind. Wir nehmen vielmehr an, dass die massenmedial beachteten Themen auch in Weblogs zu finden sind, jedoch durch Themen ergänzt werden, die den zivilgesellschaftlichen Interessen entsprechen, aber in den Medien nur wenig Beachtung finden.

In Bezug auf die Nachrichtenfaktoren können keine eindeutigen Annahmen formuliert werden. Man kann einerseits davon ausgehen, dass Nachrichtenfaktoren als wesentliche Verkörperung der Medienlogik die massenmediale Realitätskonstruktion bestimmen. Andererseits ist zu erwarten, dass sie sich - wenn sie als allgemein-menschliche Kriterien zu betrachten sind - auch in der Realitätskonstruktion durch zivilgesellschaftliche Beobachter niederschlagen. Für die inhaltliche Erweiterung des politischen Diskurses gäbe das wenig Anlass zu Optimismus. Die erweiterten Beteiligungschancen der Zivilgesellschaft würden zu einer quantitativen Zunahme von Sprechern führen, während die Inhalte aufgrund der identischen Auswahlkriterien weitgehend unverändert blieben.

13 Damit dürften sich hier die massenmedialen Realitätskonstruktionen in den Bloginhalten wiederfinden, so dass eine von der Medienlogik abweichende, spezifisch zivilgesellschaftliche Perspektive auch in dieser Studie kaum sichtbar werden kann. 
In welchem Maße Themenschwerpunkte und Nachrichtenfaktoren sich zwischen politischen Weblogs und der massenmedialen Berichterstattung unterscheiden, wird in einer quantitativen Inhaltsanalyse konventioneller Printmedien, einer reinen OnlineZeitung ${ }^{14}$ sowie politischer Weblogs in einem insgesamt zweiwöchigen Untersuchungszeitraum vom Frühjahr 2008 (21.02.2008-05.03.2008) untersucht. Untersuchungsgegenstand ist die Politikberichterstattung in der Frankfurter Allgemeinen Zeitung (FAZ) und der Süddeutschen Zeitung $(\mathrm{SZ})^{15}$, in der Netzeitung ${ }^{16}$, sowie politische Blogeinträge („postings“) aus den sieben ausgewählten politik-orientierten und gleichzeitig im Netz häufig verlinkten („incoming links“) Weblogs Schockwellenreiter ${ }^{17}$, Achse des Guten, Mein Parteibuch, Politically Incorrect Neres, Fefes Blog, Politikblog und Spreeblick. Als politisch wurden alle Beiträge gewertet, die politische Akteure oder Bezugnahmen auf staatliches Handeln enthielten. Die Themenkategorien sowie die Kategorisierung der geographischen Schwerpunkte wurden theoriegeleitet entwickelt und dann anhand des empirischen Materials ergänzt. Als Nachrichtenfaktoren wurden Schaden, Nutzen, Faktizität, Einfluss, Prominenz, Dauer, Reichweite, Konflikt und Personalisierung erfasst. $^{18}$

Die Fragestellung wird inhaltsanalytisch in zwei Schritten bearbeitet. Im Mittelpunkt des ersten Schritts stehen die thematischen und geographischen Schwerpunkte der Untersuchungseinheiten. Dieser Analyse liegt ein einwöchiger Untersuchungszeitraum (21.02.2008-27.02.2008) zugrunde. Der Zeitraum reichte aus, um hinreichende Varianz zu erzeugen. Der zweite Schritt vertieft den ersten Analyseschritt insofern, als hier die Auswahl- und Darstellungskriterien unterbalb der Themenebene untersucht werden. Dazu werden zu sieben ausgewählten Themen ${ }^{19}$ die Nachrichtenfaktoren in den drei Angebotstypen Printzeitungen, Netzeitung und Weblogs erfasst. Der Analyse der Zeitungen liegen alle themenspezifischen Artikel des Untersuchungszeitraums, der Analyse der Weblogs alle themenspezifischen Einträge in den Top 50 Weblogs der deutschen Blog Charts vom 27.02.2008 zugrunde. Der Untersuchungszeitraum umfasst zwei Wochen (21.02.2008-05.03.2008). Die Intercoderreliabilität auf der Basis von sechs Paar-

14 Die Berücksichtigung der Online-Zeitung diente der Kontrolle, ob die Unterschiede zwischen den Angebotsformen auf die Professionalität der Autoren (Print- und Online-Zeitung vs. Weblogs) oder auf Spezifika im Online-Bereich zurückgeführt werden können (Online-Zeitung und Weblogs vs. Print).

15 Montag bis Samstag, jeweils der Mantel ohne Panorama/Vermischtes, Werbung, Leserbriefe, Pressestimmen, aktuelles Lexikon, Inhaltsverzeichnis, kurze Meldungen auf S. 1, die andere Ressorts betrafen als Politik.

16 Montag bis Sonntag, dabei jeweils die aktuellste Fassung (Abruf jeweils zwischen 11 und 12 Uhr). Alle Beiträge, die unter „Politik“ und „Ansichtssache“ erschienen, sofern die Meinungsbeiträge in den Bereich Politik fielen.

17 Dieser Blog wurde zunächst bei einem zweitägigen Test als politischer Blog identifiziert, er enthielt allerdings im nachfolgenden Zeitraum kaum mehr politische Beiträge.

18 Nicht berücksichtigt wurden Ortsstatus, Überraschung und Themenetablierung. Diese Faktoren wiesen entweder - aufgrund des engen Ereignishorizonts - eine zu geringe Varianz auf (so etwa Ortsstatus und Überraschung) oder sie waren im Rahmen des Untersuchungszeitraums nicht zu ermitteln, weil keine Möglichkeit bestand, die Vorberichterstattung zu untersuchen (Themenetablierung).

19 Die Festlegung der sieben Themen erfolgte entlang der häufig genannten Themen in den laut Blogcharts meistverlinkten Blogs (Top 100 Blogs vom 27.02.2008), die regelmäßig Bezug auf politische Themen nehmen. Durch die Orientierung an den Themen der Weblogs wurde verhindert, dass für die massenmediale Berichterstattung keine Pendants in den Weblogs vorliegen (vgl. die Kurzbeschreibungen der Themenbereiche im Anhang). 
vergleichen bei vier Codierern liegt für die Identifikationsvariablen bei 1,0, für die Darstellungs-Variablen (etwa Genre, Umfang, Verweise auf andere Quellen) bei 0,85 und für die Nachrichtenfaktoren bei $0,69^{20}$ und ist damit noch zufriedenstellend.

\section{Befunde}

\subsection{Differenzielle Themen-Aufmerksamkeit und geographische Schwerpunkte}

Der erste Untersuchungsschritt nimmt alle in der ersten Woche des Untersuchungszeitraums angesprochenen politischen Themen in den Blick und beruht auf insgesamt 928 Beiträgen aus Printmedien, Netzeitung und Weblogs. Der Großteil der Beiträge findet sich dabei in FAZ und SZ $(\mathrm{N}=610)$. An zweiter Stelle stehen die sieben ausgewählten Weblogs ( $\mathrm{N}=195)$, während die Netzeitung $(\mathrm{N}=123)$ trotz des unbegrenzt verfügbaren Platzes die wenigsten politischen Beiträge im einwöchigen Untersuchungszeitraum aufweist. Es zeigen sich zwar ähnliche Topthemen (stark beachtete Themen mit über fünf Prozent Häufigkeit) auf den Agenden der drei unterschiedlichen Angebotstypen, allerdings ist sowohl der Anteil der Topthemen an der Gesamtberichterstattung als auch die Verteilung der einzelnen Topthemen sehr unterschiedlich (vgl. Abbildung 1). ${ }^{21}$ Das Verhältnis zwischen den Topthemen und den sonstigen Themen erlaubt Aussagen über die Themenvielfalt. Die diesbezüglichen Unterschiede zwischen Print- und Netzeitungsangeboten zeigen, dass die Themenvielfalt nicht an der Professionalität der Anbieter festgemacht werden kann. Professionelle Print-Journalisten und Blogger weisen einen hohen Anteil an sehr kleinteiligen „sonstigen Themen“ auf und berichten somit ähnlich vielfältig. Die professionell-journalistische Netzeitung hingegen fokussiert stärker auf die politischen Topthemen.

\section{Abbildung 1: Themenverteilung in den Angebotstypen}
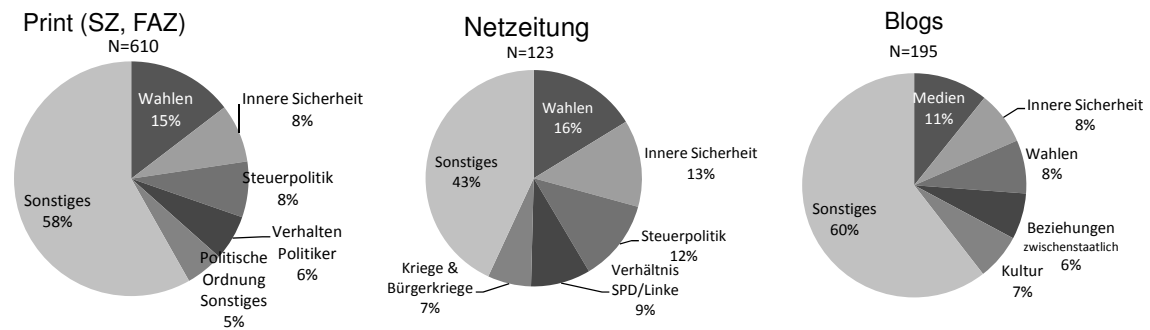

Setzen die professionellen Angebote in Bezug auf die Vielfalt unterschiedliche Prioritäten, so ist die Themenagenda von Print- und Netzeitung im Bereich der Topthemen recht ähnlich. „Wahlen“, „innere Sicherheit“ und „Steuerpolitik“ sind die Top 3-Themen der professionellen Journalisten. Die Abweichung der Netzeitung von den Print-Angeboten bei den Themen „Verhältnis SPD/Linke“ und „Kriege \& Bürgerkriege“ lässt sich durch die stärker auf Aktualität bedachte Berichterstattung der Online-Zeitung erklären. Viele

20 Damit ist ein wesentlicher methodischer Befund der Studie angesprochen. Die typischerweise extrem kurzen Blog-Einträge waren ungewöhnlich schwer zu codieren, da der Kontext für die Einschätzung der nachrichtenfaktor-relevanten Informationen fehlte.

21 Die drei Angebotstypen unterscheiden sich in Bezug auf die Themenhäufigkeiten signifikant $(\mathrm{p}<.01)$. 
kurze Meldungen erscheinen hier stündlich neu, während die einmal täglich erscheinenden Zeitungen diese Meldungen in einem Artikel zusammenfassen.

Weblogs weichen von der Themenagenda der professionellen Angebote deutlich ab. Zwar thematisieren sie ebenfalls „Wahlen“ und „innere Sicherheit“, ihr Fokus liegt allerdings auf der „Medien“-Beobachtung. Das beinhaltet vor allem die Medienkritik, also die Korrektur von Meldungen oder das Aufzeigen von Fehlern in der Berichterstattung. ${ }^{22} \mathrm{Da}$ Weblogs häufig als Resonanzraum der Massenmedien (vgl. z. B. Neuberger et al. 2007) betrachtet werden, ist das kaum überraschend. Die Rede vom Resonanzraum bedeutet allerdings nicht, dass Weblogs die massenmediale Agenda eins zu eins widerspiegeln. Nicht alle Themen ziehen die Aufmerksamkeit der Blogger gleichermaßen auf sich. An das komplexe und massiv in den etablierten Medien vertretene Thema "Steuerpolitik“ etwa wagen sich Blogger seltener als ihre professionellen Kollegen heran. Umgekehrt greifen Blogger mit der „Kultur“ ein Thema auf, das in den politischen Beiträgen der Massenmedien deutlich weniger Aufmerksamkeit findet. Weblogs reflektieren also durchaus die Aufmerksamkeitsstruktur der massenmedialen Agenda, zeigen allerdings etwas andere Prioritäten und ergänzen diese Agenda durch Nischenthemen (vgl. auch Schmidt et al. 2006: 43), die in den klassischen Medien vergleichsweise selten aufgegriffen werden.

Abbildung 2: Top 5 der geographischen Schwerpunkte der Angebotstypen im Vergleich

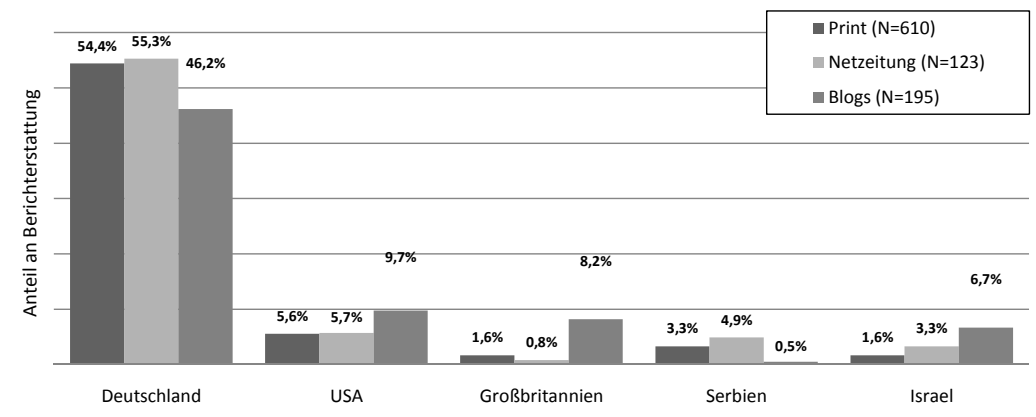

Die weblog-spezifische Vorliebe für die „Nische“ bei insgesamt moderater Abweichung von den professionellen Kollegen wird auch beim Vergleich der geographischen Schwerpunkte deutlich (vgl. Abbildung 2). ${ }^{23}$ Zwar werden Deutschland und die USA prozentual in allen drei Angebotstypen am häufigsten als Ereignisort genannt, im Hinblick auf die Anteile dieser Länder an der Berichterstattung sind aber zwischen professionellem und bürgerjournalistischem Angebot deutliche Unterschiede auszumachen. Deutschland, der unangefochtene Spitzenreiter bei den Ländernennungen, wird in mehr als der

22 Die medienkritischen Beiträge zu einem bestimmten Ereignis wurden nicht diesem zugeordnet, sondern dem Themenkomplex Medien, da die Medienkritik im Vordergrund stand.

23 Die drei Angebotstypen unterscheiden sich signifikant in Bezug auf die geographischen Schwerpunkte $(\mathrm{p}<.01)$. 
Hälfte der politischen Berichterstattung der Massenmedien thematisiert. In den Weblogs wird erstaunlicherweise seltener über Deutschland berichtet. Der lebensweltliche Nahraum, den wir in Abschnitt 4 als zivilgesellschaftliche Präferenz unterstellt haben, ist also nicht notwendig geographisch zu verstehen. Vielmehr richten Blogger ihre Aufmerksamkeit auf internationale Themen und Ereignisse, vor allem im englischen Sprachraum. Über die USA wird in den Weblogs prozentual fast doppelt so häufig berichtet wie in FAZ, SZ und Netzeitung. Noch deutlicher wird der Unterschied bei Großbritannien, das im Angebotstyp Weblog eines der meistbeachteten Länder darstellt, in den Massenmedien jedoch im Untersuchungszeitraum verhältnismäßig selten thematisiert wird. Es ist $\mathrm{zu}$ vermuten, dass dies vor allem an der Dominanz der englischen Sprache in der internationalen Blogosphäre liegt und nicht an einem generell größeren Interesse der Blogger am internationalen politischen Geschehen. Dafür spricht jedenfalls die im Vergleich mit den etablierten Massenmedien geringe Beachtung des serbisch-kosovarischen Konflikts in den Weblogs. ${ }^{24}$

\subsection{Nachrichtenfaktoren in den drei Angebotstypen}

Der zweite Untersuchungsschritt ermittelt die Konstruktionskriterien innerhalb von sieben ausgewählten Themen im gesamten Untersuchungszeitraum. Die vertiefende Analyse beruht auf 539 Beiträgen. Davon stammen 337 aus der Printberichterstattung von FAZ und SZ, 111 aus der Netzeitung und 91 aus Weblogs. Während sich fast die Hälfte der Beiträge (48 \%) mit den Koalitionsbildungen im Kontext der Landtagswahlen befasst und der Steuerskandal mit knapp 20 Prozent an zweiter Stelle steht, machen die anderen fünf Themen nur bis zu elf Prozent der Gesamtberichterstattung zu allen sieben Themen aus („Wahlen USA“ $11 \%$, „Kosovokrise“ $10 \%$, „Irak-Türkei-Konflikt“ 5 \%, „Skandal um den Begriff der Kindstötung“ und „Bergbau im Saarland“ je 4 \%). Die Themen verteilen sich relativ gleichmäßig auf die Angebotstypen. Es besteht kein signifikanter Zusammenhang zwischen Thema und Angebotstyp, so dass die Analyse über alle sieben Themen gemeinsam erfolgen kann.

Der Vergleich der Nachrichtenfaktoren in den drei Angebotstypen zeigt, welche relative Bedeutung den Nachrichtenfaktoren im professionellen Journalismus (in konventionellen Printmedien und in Online-Angeboten) und im Bürgerjournalismus zukommt. Je höher die Ausprägung eines Nachrichtenfaktors im Vergleich der Angebotstypen, desto mehr Bedeutung kommt dem Faktor im jeweiligen Angebotstyp zu. ${ }^{25}$ So hat etwa der Faktor Nutzen/Erfolg in der Netzeitung die größte Bedeutung (Mittelwert bei .31), in den Weblogs die geringste (.14).

Es zeigen sich signifikante Unterschiede für die Faktoren Nutzen/Erfolg, Faktizität, Einfluss, Prominenz und Konflikt (vgl. Tabelle 1). ${ }^{26}$ In Weblogs kommt den Faktoren

24 Das Interesse der Blogger für den israelisch-palästinensischen Konflikt widerspricht dieser Auslegung insofern nicht, als sich hier möglicherweise die Auswahl der Blogs niederschlägt: „Politically Incorrect News“ und „Die Achse des Guten“ sind deutlich pro-israelisch ausgerichtet.

25 Die Nachrichtenfaktoren sind mit zwei bzw. drei Ausprägungen operationalisiert, da sich in Vorstudien gezeigt hatte, dass nur so eine zufriedenstellende Intercoderreliabilität zu erzielen war. Wegen der unterschiedlichen Anzahl von Ausprägungen sind keine Aussagen über die relative Bedeutung der Faktoren möglich.

$26 \mathrm{Da}$ Thema und Angebotstyp statistisch unabhängig sind, kann der Befund nicht alternativ durch eine unterschiedliche Beachtung der sieben Themen in den drei Angebotstypen erklärt werden. 
jeweils die geringste Bedeutung zu, während sich die Beiträge innerbalb des professionellen Journalismus trotz der unterschiedlichen Platzrestriktionen nur wenig unterscheiden. Die Netzeitung lässt sich dabei auf der Basis dieser Befunde keineswegs als „Kompromiss“ zwischen konventioneller Printberichterstattung und Weblogs charakterisieren. Vielmehr weicht die Netzeitung noch stärker als die Printzeitungen von den Weblogs ab, indem sie Nachrichtenfaktoren die insgesamt höchste Bedeutung zuweist. Eine naheliegende Erklärung ist der hohe Anteil an kurzen Meldungen in diesem Angebotstyp.

\section{Tabelle 1: Unterschiede im Niveau der Nachrichtenfaktoren zwischen den Angebotstypen}

\begin{tabular}{lrrrr}
\hline $\begin{array}{l}\text { Nachrichtenfaktoren } \\
\text { (Ausprägungen in Klammern*) }\end{array}$ & $\begin{array}{r}\text { Print } \\
\text { N=337 } \\
\text { MW }\end{array}$ & $\begin{array}{r}\text { Netzeitung } \\
\text { N=111 } \\
\text { MW }\end{array}$ & $\begin{array}{r}\text { Weblogs } \\
\text { N=91 } \\
\text { MW }\end{array}$ & Signifikante Unterschiede*** \\
\hline Schaden (0-2) & .52 & .54 & .51 & nein \\
Nutzen/Erfolg (0-2) & .27 & .31 & .14 & ja \\
Faktizität (0-2) & .95 & 1.08 & .64 & ja \\
Einfluss (0-3) & 1.78 & 2.03 & 1.60 & ja \\
Prominenz (0-3) & 1.93 & 2.13 & 1.24 & ja \\
Dauer (0-3) & 2.17 & 2.13 & 1.93 & nein \\
Reichweite (0-3) & 2.11 & 2.07 & 1.93 & nein \\
Konflikt (0-2) & .72 & .81 & .46 & ja \\
Personalisierung (0-2) & .92 & 1.06 & .82 & nein \\
\hline
\end{tabular}

* Die Nachrichtenfaktoren in jedem Beitrag wurden in mehreren Intensitätsstufen erfasst. Der Faktor Einfluss etwa weist drei Ausprägungen auf. Die „0“ zeigt an, dass die genannten Akteure keinen Einfluss haben (zivilgesellschaftliche Akteure ohne Ämter), die „1“ bedeutet geringer Einfluss (Akteure auf kommunaler Ebene), die „2“ großer Einfluss (Landesebene sowie Massenmedien, mittlere Wirtschaftsunternehmen, Universitäten, Verbände) und die „3“ größter Einfluss (Bundespolitik und internationale Ebene; auch Freiheitsbewegungen, Banken, religiöse Zentralinstanzen). Einige Faktoren wurden lediglich mit Ausprägungen zwischen „o“ und „2“ erfasst, da sich für mehr Ausprägungen zu geringe Übereinstimmungen zwischen den Codierern gezeigt hatten.

$\because *$ Kruskal-Wallis-Test über gruppierte Mediane, Signifikanz-Niveau: 5 Prozent.

In Bezug auf die Frage nach den Spezifika zivilgesellschaftlicher Realitätskonstruktionen sind mit der vergleichsweise geringen Bedeutung der fünf Nachrichtenfaktoren Nutzen/ Erfolg, Faktizität, Einfluss, Prominenz und Konflikt in Weblogs die zentralen Befunde der Untersuchung markiert. Im Bürgerjournalismus werden im Gegensatz zum professionellen Journalismus weniger positive Ereignismerkmale akzentuiert (Nachrichtenfaktor Nutzen/Erfolg). Die Blogbeiträge beziehen sich ferner seltener auf faktisches Geschehen, sondern räumen Stellungnahmen und Vermutungen mehr Raum ein (Faktizität). Ebenso werden weniger einflussreiche (Einfluss) und bekannte (Prominenz) Akteure angesprochen als im professionellen Journalismus, und die Realitätskonstruktionen sind weniger konfliktträchtig (Konflikt). Einige der Erwartungen an zivilgesellschaftliche Realitätskonstruktionen lassen sich mit den Befunden bestätigen: Nicht Einfluss und Prominenz entscheiden über den Zugang von Akteuren zur Öffentlichkeit, sondern auch Bürger ohne diese Merkmale erhalten im Bürgerjournalismus Beachtung. Auch die geringe Bedeutung von Konflikt - ein häufig kritisiertes Element massenme- 
dialer Dramatisierung - deutet darauf hin, dass hier die Medienlogik außer Kraft gesetzt ist.

Das betrifft allerdings keineswegs alle Nachrichtenfaktoren. Die Gemeinsamkeiten zwischen den professionellen und nicht-professionellen Angebotstypen in Bezug auf die vier Faktoren Schaden, Dauer, Reichweite und Personalisierung zeigen, dass die zivilgesellschaftliche Realitätskonstruktion nicht grundsätzlich von der Medienlogik abweicht. Diese Faktoren leiten in allen Angebotstypen gleichermaßen die Realitätskonstruktion. Einige Befunde enttäuschen zu optimistische Erwartungen an eine zivilgesellschaftliche Realitätskonstruktion: Negativen Realitätsaspekten (Schaden) wird von Bürgerjournalisten ebenso viel Bedeutung zugewiesen wie von professionellen Journalisten, punktuellen Ereignissen wird Vorrang vor möglicherweise mitteilungswürdigeren langfristigen Entwicklungen (Dauer) eingeräumt und personalisierte Darstellungen werden abstrakten Sachverhalten (Personalisierung) gegenüber bevorzugt. Insgesamt weichen die Realitätskonstruktionen im Bürgerjournalismus also nicht in jeder Hinsicht von den Konstruktionen im professionellen Journalismus ab. Vielmehr ergeben sich ausgerechnet in Bezug auf häufig kritisierte Faktoren wie Schaden, Dauer und Personalisierung Übereinstimmungen mit der Berichterstattung der etablierten Massenmedien. Eine genuin zivilgesellschaftliche Perspektive auf das politische Geschehen wird hier nicht durchgehend deutlich.

\section{Fazit}

Ausgangspunkt der Studie war die Frage nach dem inhaltlichen Beitrag von politischen Weblogs zum politischen Diskurs in Deutschland. Die aus der aktiven Beteiligung der Zivilgesellschaft resultierenden Konsequenzen für die Realitätskonstruktionen dienen daher als Kriterium, wenn es darum geht, den Nutzen von Weblogs für die politische Öffentlichkeit abzuschätzen. Trotz des explorativen Charakters der Studie wurde mit der vergleichenden Inhaltsanalyse zwischen politischen Weblogs und professionelljournalistischen Print- und Online-Zeitungen die bislang meist theoretische und ideologische Debatte empirisch bereichert. Es galt, die mögliche Erweiterung der inhaltlichen Vielfalt durch politische Weblogs zu erfassen und zu klären, ob hier grundsätzlich andere, von der häufig kritisierten Medienlogik abweichende Auswahl- und Darstellungskriterien angewandt werden.

Die Befunde lassen den Schluss zu, dass politische Weblogs die Berichterstattung der etablierten Massenmedien zwar durch häufige Bezugnahmen reflektieren, diese aber auch ergänzen, indem sie zusätzliche Themen aufgreifen und teilweise andere geographische Schwerpunkte setzen. Politische Weblogs erweitern damit den politischen Diskurs wahrnehmbar. In Bezug auf die geltenden Auswahl- und Darstellungskriterien innerhalb einzelner Themen zeigt sich ein widersprüchliches Bild. Während sich einige Nachrichtenfaktoren als rein professionelle Kriterien erweisen, bestimmen andere auch die zivilgesellschaftlichen Realitätskonstruktionen. Nur teilweise entsprechen damit die Befunde den Erwartungen an eine spezifisch zivilgesellschaftliche Realitätskonstruktion. Vor allem im Hinblick auf die geringe Bedeutung der Faktoren Einfluss und Prominenz ergeben sich hier Hinweise auf die gewünschte genuin zivilgesellschaftliche Perspektive. Dagegen stehen allerdings die Faktoren Schaden, Dauer und Personalisierung, die auch die zivilgesellschaftlichen Realitätsbilder prägen. Diese Befunde bedeuten eine gewisse Ernüchterung in Bezug auf die optimistischen Szenarien zur Zukunft des Web 2.0. Der Bürgerjournalismus erweist sich hier nicht als der „bessere“ Journalismus.

Ernüchterung stellt sich allerdings nur dann ein, wenn man die Nachrichtenfaktoren als rein journalistische Konstruktionskriterien versteht. Begreift man dagegen Nach- 
richtenfaktoren als allgemeine, komplexitätsreduzierende Mechanismen menschlicher Wahrnehmung, die nicht per se zu einem Realitätsbild führen, das für den demokratischen Prozess unnütz oder gar schädlich ist, so sind die Gemeinsamkeiten zwischen Bürgerjournalismus und professionellem Journalismus kaum überraschend. Wichtig erscheint in diesem Zusammenhang eine Differenzierung zwischen den einzelnen Nachrichtenfaktoren und eine Berücksichtigung der jeweiligen Kontexte. Während einige Nachrichtenfaktoren durchaus negative Konsequenzen für das entstehende Politikbild haben können, stehen andere - in bestimmten Kontexten - für eine sinnvolle Aufmerksamkeitssteuerung.

Die Ernüchterung in Bezug auf die doch relativ große Ähnlichkeit zwischen zivilgesellschaftlicher und professionell-journalistischer Realitätskonstruktion relativiert sich noch in anderer Hinsicht. Es ist davon auszugehen, dass die Realitätskonstruktionen in politischen Weblogs sich zwar kaum durch Nachrichtenfaktoren, aber durch andere, hier nicht erfasste inhaltliche Merkmale (etwa subjektive Bedeutsamkeit, Authentizität, Meinungsfreude und Humor) klar von massenmedialer Berichterstattung unterscheiden und hier auch mehr Anlass zur Hoffnung auf eine Belebung des politischen Diskurses besteht. Nachrichtenfaktoren lassen sich nach unseren Befunden zwar teilweise als „Blogfaktoren“ begreifen, da sie auch politische Weblogs kennzeichnen. Daneben gilt es aber, Faktoren zu identifizieren, die ausschließlich politische Weblogs kennzeichnen und nach Möglichkeit auch ihre große Attraktivität erklären können. Solche Merkmale zunächst theoretisch zu entwickeln und dann empirisch zu überprüfen, stellt die nächste Herausforderung der kommunikationswissenschaftlichen Forschung dar.

\section{Literatur}

Armborst, Matthias (2006): Kopfjäger im Internet oder publizistische Avantgarde. Was Journalisten über Weblogs und ihre Macher wissen sollten. Berlin: LIT (Recherche-Journalismus und kritische Medienpolitik, 4).

Berendt, Bettina; Schlegel, Martin; Koch, Robert (2008): Die deutschsprachige Blogosphäre: Reifegrad, Politisierung, Themen und Bezug zu Nachrichtenmedien. In: Zerfaß, Ansgar; Welker, Martin; Schmidt, Jan (Hg.): Kommunikation, Partizipation und Wirkungen im Social Web. Band 2: Strategien und Anwendungen: Perspektiven für Wirtschaft, Politik und Publizistik. Köln: Halem (Neue Schriften zur Online-Forschung, 3), S. 72-96.

Bruns, Axel (2007): Produsage. In: 6th Creativity \& Cognition Conference (Hg.): Proceedings of the 2007 Conference on Creativity and Cognition. Washington D.C., S. 99-106.

Dahlberg, Lincoln (2001): The Internet and Democratic Discourse. Exploring the Prospects of Online Deliberative Forums Extending the Public Sphere. In: Information, Communication \& Society, Jg. 4, H. 4, S. 615-633.

Dahlberg, Lincoln (2007): The Internet, Deliberative Democracy and Power: Radicalizing the Public Sphere. In: International Journal of Media and Cultural Politics, Jg. 3, H. 1, S. 47-64.

Donges, Patrick (2000): Technische Möglichkeiten und soziale Schranken elektronischer Öffentlichkeit. Positionen zur elektronischen Öffentlichkeit und ihr Bezug zu Öffentlichkeitsmodellen. In: Jarren, Otfried (Hg.):Zerfall der Öffentlichkeit. 1. Aufl. Wiesbaden: Westdt. Verl. (Reihe Mediensymposium Luzern, 6), S. 255-265.

Eilders, Christiane (1997): Nachrichtenfaktoren und Rezeption. Eine empirische Analyse zur Auswahl und Verarbeitung politischer Information. Opladen: Westdt. Verl. (Studien zur Kommunikationswissenschaft, 20).

Eilders, Christiane (2008): Medien als Produzenten öffentlicher Meinungen. Pressekommentare als Manifestation der politischen Akteursrolle. In: Pfetsch, Barbara; Adams, Silke (Hg.): Medien als politische Akteure. Konzepte und Analysen. Wiesbaden: VS-Verlag, S. 27-51. 
Eilders, Christiane (2010): Zivilgesellschaftliche Beteiligung im Medienbereich. In: Kleinsteuber, Hans; Nehls, Sabine; Voss, Kathrin (Hrsg.): Media Governance in Europe - Regulation, Participation, Co-Determination. Wiesbaden: VS-Verlag (im Erscheinen).

Eilders, Christiane; Wirth, Werner (1999): Die Nachrichtenwertforschung auf dem Weg zum Publikum: Eine experimentelle Überprüfung des Einflusses von Nachrichtenfaktoren bei der Rezeption. In: Publizistik, Jg. 44, H. 1, S. 35-57.

Engesser, Sven (2008): Partizipativer Journalismus: Eine Begriffsanalyse. In: Zerfaß, Ansgar; Welker, Martin; Schmidt, Jan (Hg.): Kommunikation, Partizipation und Wirkungen im Social Web. Band 2: Strategien und Anwendungen: Perspektiven für Wirtschaft, Politik und Publizistik. Köln: Halem (Neue Schriften zur Online-Forschung, 3), S. 47-71.

Engesser, Sven; Wimmer, Jeffrey (2009): Gegenöffentlichkeit(en) und partizipativer Journalismus im Internet. In: Publizistik, Jg. 54, H. 1, S. 43-63.

Ferree, Myra M.; Gamson, William A.; Gerhards, Jürgen; Rucht, Dieter (2002): Four Models of the Public Sphere in modern Democracies. In: Theory and Society, Jg. 31, H. 3, S. 289-324, www. ssc.wisc.edu/ mferree/documents/FerreeGamsonGerhardsRucht.pdf [12.02.2010].

Fretwurst, Benjamin (2008): Nachrichten im Interesse der Zuschauer. Eine konzeptionelle und empirische Neubestimmung der Nachrichtenwerttheorie. Konstanz: UVK.

Habermas, Jürgen (1998): Faktizität und Geltung. Beiträge zur Diskurstheorie des Rechts und des demokratischen Rechtsstaats. 3. Aufl. Frankfurt am Main: Suhrkamp.

Habermas, Jürgen (2008): Hat die Demokratie noch eine epistemische Dimension? Empirische Forschung und normative Theorie. In: Ach, Europa. Orig.-Ausg., 1. Aufl. Frankfurt am Main: Suhrkamp, S. 138-191.

Hamelink, Cees J. (2000): The Ethics of Cyberspace. London, Thousand Oaks Calif.: Sage Publications.

Heming, Ralf (1997): Öffentlichkeit, Diskurs und Gesellschaft. Zum analytischen Potential und zur Kritik des Begriffs der Öffentlichkeit bei Habermas. Wiesbaden: DUV (DUV: Sozialwissenschaft).

Herring, Susan C.; Kouper, Inna; Scheidt, Lois Ann; Wright, Elijah L. (2004): Women and Children Last: The Discursive Construction of Weblogs. Hrsg. von Laura J. Gurak. Department of Rhetoric, University of Minnesota. (Into the blogosphere: Rhetoric, community, and culture of weblogs), http://blog.lib.umn.edu/blogosphere/women_and_children.html [12.02.2010].

Hesse, Franka (2008): Die Geschlechterdimension von Weblogs: Inhaltsanalytische Streifzüge durch die Blogosphäre. In: kommunikation@gesellschaft, Jg. 9, H. 1, www.soz.uni-frank furt.de/K.G/B1_2008_Hesse.pdf [12.02.2010].

Klein, Ansgar (2008): Zivilgesellschaft und Demokratie. Ideengeschichtliche, demokratietheoretische und politisch-soziologische Zugänge. In: Forschungsjournal Neue Soziale Bewegungen, Jg. 21, H. 3, S. 189-237.

Koopmans, Ruud; Zimmermann, Anne (2003): Internet: A New Potential for European Political Communication. Paper SP IV 2003-402 presented to the International Conference on „Europeanisation of Public Spheres? Political Mobilisation, Public Communication, and the European Union“. Veranstaltung vom 20.-22-06.2003.

Lenhart, Amanda; Fox, Susannah (2006): Bloggers. A Portrait of the Internet's New Storytellers. Pew Internet \& American Life Project, www.pewinternet.org/ /media/Files/Reports/2006/ PIP\%20Bloggers\%20Report\%20July\%2019\%202006.pdf [17.02.2010].

Löffelholz, Martin (Hg.) (2004): Theorien des Journalismus. Ein diskursives Handbuch. 2., vollst. überarb. und erw. Aufl. Wiesbaden: VS Verlag.

Marschall, Stefan (1997): Politik online. Demokratische Öffentlichkeit dank Internet. In: Publizistik, Jg. 42, H. 3, S. 304-324.

Meckel, Miriam (2009): Qualitätsjournalismus: In der Grotte der Erinnerung. FAZ.net. Online verfügbar unter http://www.faz.net/s/Rub475F682E3FC24868A8A5276D4FB916D7/Doc E67F2AC3CEC9746EC93DC9FF0E5679312 ATpl Ecommon Scontent.html [17.02.2010].

Morriset, Lloyd (2003): Technologies of Freedom. In: Jenkins, Henry; Thorburn, David; Seawell, Brad (Hg.): Democracy and New Media. Cambridge Mass.: MIT Press (Media in transition), S. 21-32. 
Neidhardt, Friedhelm (1994): Öffentlichkeit, öffentliche Meinung, soziale Bewegungen. In: Neidhardt, Friedhelm (Hg.): Öffentlichkeit, öffentliche Meinung, soziale Bewegungen. Opladen: Westdt. Verl. (Kölner Zeitschrift für Soziologie und Sozialpsychologie, Sonderheft), S. 7-41.

Neuberger, Christoph (2003): Onlinejournalismus: Veränderungen - Glaubwürdigkeit - Technisierung. In: Media Perspektiven, H. 3, S.131-138, www.media-perspektiven.de/uploads/ tx_mppublications/03-2003_Neuberger.pdf [17.02.2010].

Neuberger, Christoph (2008): Internet und Journalismusforschung. Theoretische Neujustierung und Forschungsagenda. In: Quandt, Thorsten; Schweiger, Wolfgang (Hg.): Journalismus online - Partizipation oder Profession. 1. Aufl. Wiesbaden: VS Verlag, S. 17-42.

Neuberger, Christoph; Nuernbergk, Christian; Rischke, Melanie (2007): Weblogs und Journalismus: Konkurrenz, Ergänzung oder Integration. Eine Forschungssynopse zum Wandel der Öffentlichkeit im Internet. In: Media Perspektiven, H. 2, S. 96-112, www.media-perspektiven.de/ uploads/tx_mppublications/02-2007_Neuberger.pdf [17.02.2010].

Neuberger, Christoph; Nuernbergk, Christian; Rischke, Melanie (2009): Weblogs und Journalismus: Konkurrenz, Ergänzung oder Integration. Eine Forschungssynopse zum Wandel der Öffentlichkeit im Internet. In: Media Perspektiven, H. 4, S. 174-188, www.media-perspektiven.de/ uploads/tx_mppublications/04-2009_Neuberger.pdf [17.02.2010].

Niggemeier, Stefan (2009): Zukunft des Journalismus: Was würde uns fehlen ohne Journalismus. FAZ.net, www.faz.net/s/Rub475F682E3FC24868A8A5276D4FB916D7/Doc E6A454527E2 D7424284F5A51A68157376 ATpl Ecommon Scontent.html [17.02.2010].

Page, Benjamin I. (1996): Who Deliberates. Mass Media in Modern Democracy. Chicago: Univ. of Chicago Press.

Papacharissi, Zizi (2007): Audiences as Media Producers. Content Analysis of 260 Blogs. In: Tremayne, Mark (Hg.): Blogging, Citizenship, and the Future of Media. London, New York: Routledge, S. 21-38.

Quandt, Thorsten (2004): Das Ende des Journalismus. Theorien zur Analyse netzbasierter Medienkommunikation. In: Löffelholz, Martin (Hg.): Theorien des Journalismus. Ein diskursives Handbuch. 2., vollst. überarb. und erw. Aufl. Wiesbaden: VS Verlag, S. 451-468.

Quandt, Thorsten; Schweiger, Wolfgang (Hg.) (2008): Journalismus online - Partizipation oder Profession. 1. Aufl. Wiesbaden: VS Verlag.

Rucht, Dieter; Yang, Mundo; Zimmermann, Ann (2008): Politische Diskurse im Internet und in Zeitungen. Das Beispiel Genfood. 1. Aufl. Wiesbaden: VS Verlag.

Schmidt, Jan (2009): How do Blogs Comment on Mainstream Media Content? Results from a Link Analysis. Charts zum Vortrag bei der General Online Research Conference vom 08.04.2009. Wien, www.schmidtmitdete.de/archives/456 [17.02.2010].

Schmidt, Jan; Paetzolt, Matthias; Wilbers, Martin (2006): Stabilität und Dynamik von WeblogPraktiken. Ergebnisse der Nachbefragung zur „Wie ich blogge?!”-Umfrage. Bamberg. (Berichte aus der Forschungsstelle „Neue Kommunikationsmedien”, 3), www.ssoar.info/ssoar/files/ 2008/237/fonkbericht0603.pdf [17.02.2010].

Schmidt, Jan; Wilbers, Martin (2006): Wie ich blogge. Erste Ergebnisse der Weblogbefragung 2005. Bamberg. (Berichte aus der Forschungsstelle „Neue Kommunikationsmedien”, 1), www. ssoar.info/ssoar/files/2008/235/fonkbericht0601.pdf [17.02.2010].

Scholl, Armin; Weischenberg, Siegfried (1998): Journalismus in der Gesellschaft. Theorie, Methodologie und Empirie. Opladen, Wiesbaden: Westdt. Verl.

Schulz, Winfried (1976): Die Konstruktion von Realität in den Nachrichtenmedien. Analyse der aktuellen Berichterstattung. Freiburg: Alber.

Shoemaker, Pamela J.; Cohen, Akiba A. (2006): News around the World. Content, Practitioners, and the Public. New York, NY: Routledge.

Sifry, David (2006): State of the Blogosphere. Oktober 2006, www.sifry.com/alerts/archives/ 000443.html [21.02.2010].

Trammel, Kaye D. (2006): Blog Offensive: An Exploratory Analysis of Attacks Published on Campaign Blog Posts from a Political Public Relations Perspective. In: Public Relations Review, Jg. 32, H. 4, S. 402-406.

Tremayne, Mark; Zheng, Nan; Lee, Jae Kook; Jeong, Jaekwan (2006): Issue Publics on the Web: Applying Network Theory to the War Blogosphere. In: Journal of Computer-Mediated Com- 
munication, Jg. 12, H. 1, Artikel 15, http://jcmc.indiana.edu/vol12/issue1/tremayne.html [17.02.2010].

Trepte, Sabine; Reinecke, Leonard; Behr, Katharina-Maria (2008): Qualitätserwartungen und ethischer Anspruch bei der Lektüre von Blogs und von Tageszeitungen. In: Publizistik, Jg. 53, H. 4, S. 509-534, www.vsjournals.de/pdf/beitrag_publizistik_04-2008.pdf [17.02.2010].

Unger, Frank (2005): Die Blogosphäre - Inhaltliche Strukturen deutschsprachiger Weblogs. Eine Analyse von publizistischen Potenzialen und thematischen Schwerpunkten. Magisterarbeit. Dresden. TU, Kommunikationswissenschaft, http://static.twoday.net/neuronal/files/magisterarbeit.pdf [17.02.2010].

Williams, Andrew Paul; Trammell, Kaye D.; Postelnicu, Monica; Landreville, Kristen D.; Martin, Justin D. (2005): Blogging and Hyperlinking: Use of the Web to Enhance Visibility during the 2004 US Campaign. In: Journalism Studies, Jg. 6, H. 2, S. 177-186.

Xenos, Michael (2008): New Mediated Deliberation: Blog and Press Coverage of the Alito Nomination. In: Journal of Computer-Mediated Communication, Jg. 13, H. 2, S. 485-503.

Zerfaß, Ansgar; Welker, Martin; Schmidt, Jan (Hg.) (2008): Kommunikation, Partizipation und Wirkungen im Social Web. Band 1: Grundlagen und Methoden: Von der Gesellschaft zum Individuum. Köln: Halem (Neue Schriften zur Online-Forschung, 2).

Zerfaß, Ansgar; Welker, Martin; Schmidt, Jan (Hg.) (2008): Kommunikation, Partizipation und Wirkungen im Social Web. Band 2: Strategien und Anwendungen: Perspektiven für Wirtschaft, Politik und Publizistik. Köln: Halem (Neue Schriften zur Online-Forschung, 3).

\section{Anhang: Kurzbeschreibung der Themen}

Wablen USA: Alle Ereignisse rund um den Vorwahlkampf der Präsidentschaftskandidaten in den USA. Dazu gehören z. B. Ereignisse im Bereich der Wahlkampfstrategien, der TV-Duelle sowie Reaktionen auf Umfrageergebnisse.

Koalitionen in den Ländern: Ereignisse im Kontext der Landtagswahlen in Hessen und Hamburg und der zugehörigen Koalitionsbildung, insbesondere parteipolitische Ereignisse, die sich auf das Verhältnis zur Linkspartei beziehen. Ebenfalls berücksichtigt wurden berichtete Ereignisse im Zuge der Koalitionsbildung.

Steuerskandal: Alle Ereignisse, die sich im Nachgang des Steuerskandals in Liechtenstein ereigneten. Dazu zählen die Ermittlungen der Steuerfahndung, internationale Beziehungen sowie der Ankauf von gestohlenen Kundendaten einer Liechtensteiner Bank durch den BND.

Kosovo: Ereignisse im Rahmen der Unabhängigkeitsbestrebungen des Kosovo und auf die Proklamierung der Unabhängigkeit am 17.02.2008 folgende Unruhen.

Kindstötung: Der Ministerpräsident von Sachsen-Anhalt, Wolfgang Böhmer, äußerte in einem Interview mit dem Nachrichtenmagazin Focus, dass Kindstötungen in der DDR und heute in den neuen Bundesländern „für manche ein Mittel der Familienplanung sind“. Codiert wurden als Ereignisse in diesem Kontext z. B. Reaktionen auf diese Äußerung und auf die Entschuldigung Böhmers vor dem Landtag von Sachsen-Anhalt.

Bergbau: Am 23.02.2008 ereigneten sich im Saarland die bisher stärksten bergbaubedingten Erderschütterungen und es kam zu Sachbeschädigungen an Häusern. Bürger schlossen sich in den Abbaugebieten zu Interessensgemeinschaften zusammen und forderten einen Ausstieg aus dem Bergbau, welcher unter verschiedenen Perspektiven von den Politikern diskutiert wurde. Der Bergbau im Saarland wurde daraufhin eingestellt.

Irak und Türkei: Das türkische Militär marschierte im Nordirak ein, um Rebellen der PKK zu bekämpfen, die von dort aus operierten. Codiert wurden Artikel über die Er- 
M\&K 58. Jahrgang 1/2010

eignisse im Nordirak sowie Pressestimmen zur Vorgehensweise des türkischen Militärs und zur Situation der PKK. 